\title{
Disturbances of Ligand Potency and Enhanced Degradation of the Human Glycine Receptor at Affected Positions G160 and T162 Originally Identified in Patients Suffering from Hyperekplexia
}

\author{
Sinem Atak ${ }^{1}$, Georg Langlhofer ${ }^{1}$, Natascha Schaefer ${ }^{1}$, Denise Kessler ${ }^{1}$, \\ Heike Meiselbach ${ }^{2}$, Carolyn Delto ${ }^{3}$, Hermann Schindelin ${ }^{3}$ and Carmen Villmann ${ }^{1 *}$ \\ ${ }^{1}$ Institute for Clinical Neurobiology, Julius-Maximilians-University of Würzburg, Würzburg, Germany, ${ }^{2}$ Bioinformatics \\ Department, Institute of Biochemistry, Friedrich-Alexander-University Erlangen-Nürnberg, Erlangen, Germany, ${ }^{3}$ Rudolf \\ Virchow Center for Experimental Biomedicine, Würzburg, Germany
}

OPEN ACCESS

Edited by:

Detlev Boison

Legacy Research Institute, USA

Reviewed by:

Maria José Diógenes, University of Lisbon, Portugal

Nicole Déglon,

Centre Hospitalier Universitaire

Vaudois, Switzerland

*Correspondence:

Carmen Villmann

Villmann_C@ukw.de

Received: 10 September 2015 Accepted: 04 December 2015 Published: 22 December 2015

Citation:

Atak S, Langlhofer G, Schaefer N, Kessler D, Meiselbach H, Delto C, Schindelin H and Villmann C (2015) Disturbances of Ligand Potency and Enhanced Degradation of the Human Glycine Receptor at Affected Positions G160 and T162 Originally Identified in Patients Suffering from Hyperekplexia.

Front. Mol. Neurosci. 8:79. doi: 10.3389/fnmol.2015.00079
Ligand-binding of Cys-loop receptors is determined by N-terminal extracellular loop structures from the plus as well as from the minus side of two adjacent subunits in the pentameric receptor complex. An aromatic residue in loop B of the glycine receptor (GlyR) undergoes direct interaction with the incoming ligand via a cation- $\pi$ interaction. Recently, we showed that mutated residues in loop B identified from human patients suffering from hyperekplexia disturb ligand-binding. Here, we exchanged the affected human residues by amino acids found in related members of the Cys-loop receptor family to determine the effects of side chain volume for ion channel properties. GlyR variants were characterized in vitro following transfection into cell lines in order to analyze protein expression, trafficking, degradation and ion channel function. GlyR $\alpha 1$ G160 mutations significantly decrease glycine potency arguing for a positional effect on neighboring aromatic residues and consequently glycine-binding within the ligand-binding pocket. Disturbed glycinergic inhibition due to T162 $\alpha 1$ mutations is an additive effect of affected biogenesis and structural changes within the ligand-binding site. Protein trafficking from the ER toward the ER-Golgi intermediate compartment, the secretory Golgi pathways and finally the cell surface is largely diminished, but still sufficient to deliver ion channels that are functional at least at high glycine concentrations. The majority of T162 mutant protein accumulates in the ER and is delivered to ER-associated proteasomal degradation. Hence, G160 is an important determinant during glycine binding. In contrast, T162 affects primarily receptor biogenesis whereas exchanges in functionality are secondary effects thereof.

Keywords: Cys-loop receptor, glycine receptor, loop B, side chain properties, ligand potencies, hyperekplexia

Abbreviations: GlyR, glycine receptor; wt, wild type; ECD, extracellular domain; TM, transmembrane; ER, Endoplasmic reticulum; Cys, cysteine. 


\section{INTRODUCTION}

Glycine receptors (GlyRs) are heteropentameric ligand-gated ion channels and belong into the superfamily of Cys-loop receptors (Lynch, 2004). Glycinergic disinhibition based on GlyR mutations is associated with neuromotor deficiencies (Schaefer et al., 2013). The immunoglobulin-like structure of the GlyR $\mathrm{N}$-terminus is determined by a short $\alpha$-helix and $10 \beta$-sheets connected by loop structures forming the large extracellular domain (ECD) followed by four transmembrane domains (TM14) and a short C-terminus (Du et al., 2015; Huang et al., 2015; Moraga-Cid et al., 2015). The ECD harbors the agonist and antagonist binding sites formed by loops A, B, C from one subunit and loops E, F, G from an adjacent subunit (Brams et al., 2011; Yu et al., 2014). The inhibitory GlyR complex is formed by three $\alpha$ subunits $(\alpha 1, \alpha 2, \alpha 3)$ and a single $\beta$ subunit arranged in a $2 \alpha: 3 \beta$ configuration (Grudzinska et al., 2005).

Glycinergic inhibition is most important in adult brain stem and spinal cord mediating processes such as motor control, pain sensitization and respiratory rhythm. Concerning the nerve-muscle circuit, GlyRs are postsynaptically expressed in the membrane of motoneurons. Upon glycine-release from neighboring inhibitory interneurons GlyRs get activated and a chloride ion influx leads to hyperpolarization of the motoneurons, balancing excitation, and consequently muscle contraction and relaxation (Rajendra et al., 1997).

Mutations in the GlyR a1 subunit gene GLRA1 are the most common cause for the rare neuromotor disorder hyperekplexia (Stiff baby syndrome, Startle disease, OMIM 149100). Typical symptoms are neonatal hypertonia and exaggerated startle response observed shortly after birth. Several mutations associated with hyperekplexia have been detected all over the GlyR $\alpha 1$ sequence. Most dominant mutants cluster in TM2 forming the ion channel domain and adjacent loop structures with some exceptions. Recessive mutations are distributed over the entire $\alpha 1$ sequence (Harvey et al., 2008; Schaefer et al., 2013; Bode and Lynch, 2014). A previous classification of dominant mutants affecting channel function and recessive mutants disrupting receptor biogenesis has recently been specified by defective neuronal subcompartimental trafficking. This study concentrated on GlyR loop B (G160R, T162M) and loop D (W68C, D70N, R72H) residues (Schaefer et al., 2015). Translational approaches based on human mutations from patients identified important GlyR residues associated with ligand-binding, conformational changes, ion channel gating, opening, desensitization, and trafficking (Saul et al., 1999; Villmann et al., 2009; Chung et al., 2010; Bode and Lynch, 2013; Bode et al., 2013). The process of ligand-binding is not only mediated by residues within the binding site, but also by the small extracellular loop between TM2-3. The underlying mechanism is thought to involve conformational rearrangements further down in the structure (Maksay et al., 2008; Pless and Lynch, 2009; Lape et al., 2012). Other residues such as P250 localized in loop TM1-2 increase receptor desensitization by altering TM positioning after ion channel opening (Saul et al., 1999; Breitinger et al., 2001). Furthermore, affected arginine residues at the $\mathrm{N}$-terminal end of transmembrane helices
(R252 before TM2 and R392 lining TM4) are important start or stop signals for TM helices and therefore most probably interfere with receptor membrane integration. Other residues in recessive hyperekplexia (S231 and I244) localized in TM1 have only marginal effects on receptor biogenesis. The observed decrease in amount of receptor at the cell surface is still sufficient to form functional channels (Vergouwe et al., 1999; Villmann et al., 2009). A recent study on mutations in loop $\mathrm{D}$, which is opposed to the protein surface, showed a largely diminished receptor trafficking due to accumulation of the majority of mutated receptor protein in the ER compartment. Based on a cellular lack of quality control in the ER, receptor subpopulations are able to cycle toward the secretory pathways of Golgi compartments and finally reach the cell surface. The numbers of surface receptors, however, were insufficient to enable functional ion channel formations (Schaefer et al., 2015). Although, the X-ray structure of GlyRs has been recently solved (Du et al., 2015; Huang et al., 2015), domain analysis will help to understand the molecular processes of neurotransmitter binding, translation of ligand-binding into channel opening and receptor closure as well as to describe the pathomechanisms in affected patients.

Here, we analyzed the recently discovered human mutations G160R and T162M in loop B of the ECD and exchanged the affected residues by other amino acids with respect to differences in side chain volume and charge at amino acid positions G160 and T162 close to the glycine-binding site. Our aim was to understand the importance of both residues within the aromatic net shaping the conformation of the neurotransmitterbinding site. We introduced at position G160 an alanine and a serine present in other Cys-loop receptors at the corresponding amino acid position. Both residues, G160 and T162, carry small and uncharged side chains compared to the positively charged arginine and the methionine carrying a hydrophobic side chain identified in hyperekplexia patients. At position T162 we converted threonine into a negatively charged aspartic acid (present in $\mathrm{GABA}_{\mathrm{C}} \pi$ and $\mathrm{AChR} \alpha 5$ ), an asparagine able to form hydrogen bond interactions with the peptide backbone (similar to ELIC), and proline providing conformational rigidity (present in $\mathrm{GABA}_{\mathrm{A}} \gamma 2$ ). The main focus of this study was on ligandefficacy and receptor trafficking. Whereas position 160 primarily leads to decreased ligand potency, lowered agonist potency is a secondary effect for mutations at amino acid position 162 . Changes at residue 162 located at the C-terminal end of loop $\mathrm{B}$ result in ER accumulation with $50 \%$ or less receptor leaving the ER toward the cell surface and the formation of functional ion channels with highly disturbed agonist efficacies. Thus, the appropriate sequence organization of loop B is a key component for the conformation of the neurotransmitter binding-pocket of the inhibitory GlyR.

\section{MATERIALS AND METHODS}

\section{Site-Directed Mutagenesis}

All mutations were introduced by PCR using primers carrying the appropriate mutation at the desired position (Invitrogen, 
Darmstadt, Germany). Amplimers carrying the mutations were digested with restriction enzymes for cloning (Xho I and Hind III). As parental clone GlyR $\alpha 1$ cDNA in the vector pRK5 was used. After digestion with Xho I and Hind III the PCR products were subcloned into GlyR a1 wild type (wt). All mutations were verified by sequencing (LGC Genomics, Berlin, Germany).

\section{Cell Lines}

HEK293 cells (Human embryonic kidney cells) were purchased from ATCC and grown in Earle's minimal essential medium (MEM) supplemented with $10 \%$ fetal calf serum, $200 \mathrm{mM}$ GlutaMAX, $100 \mathrm{mM}$ sodium pyruvate and $50 \mathrm{U} / \mathrm{mL}$ penicillin/streptomycin (Sigma-Aldrich, St. Louis, MO, USA) under standard growth conditions at $37^{\circ} \mathrm{C}$ and $5 \% \mathrm{CO}_{2}$. COS7 cells (African green monkey kidney cells) were purchased from ATCC and grown in Dulbecco's modified Earle's medium (DMEM) with the same supplements added for HEK293 cells and under the same growth conditions.

\section{Transfection}

HEK293 cells were transiently transfected using a modified calcium-phosphate precipitation method, were a mixture of plasmid DNA, $\mathrm{CaCl}_{2}$, and 2x HBS buffer (50 mM HEPES, $12 \mathrm{mM}$ glucose, $10 \mathrm{mM} \mathrm{KCl}, 280 \mathrm{mM} \mathrm{NaCl}, 1.5 \mathrm{mM} \mathrm{Na}_{2} \mathrm{HPO}_{4}$ ) was applied onto the cells.

COS7 cells were transfected with DNA diluted in PBS plus $10 \mathrm{mg} / \mathrm{mL}$ DEAE-dextran (Sigma-Aldrich, St. Louis, MO, USA). After 30 min of incubation under standard conditions, media was aspirated and fresh media containing $10 \mathrm{mM}$ chloroquine was applied for $3 \mathrm{~h}$. All experiments were done $24-48 \mathrm{~h}$ posttransfection.

\section{Biotinylation of Cell Surface Protein}

The biotinylation assay was performed on HEK293 cells transiently expressing the desired GlyR $\alpha 1$ (wt) or $\alpha 1$ variants. Cells were plated on $10 \mathrm{~cm}$ dishes. $48 \mathrm{~h}$ after transfection, medium was removed and cells were washed three times with ice-cold PBS (GE Healthcare, Freiburg, Germany). The surface proteins were labeled by incubating the cells for $30 \mathrm{~min}$ with 1 $\mathrm{mg} / \mathrm{mL}$ EZ-Link Sulfo-NHS-LC-biotin (sulfonosuccinimidyl-6(biotin-amido)-hexanoate (Pierce Biotechnologies, Rockford, IL, USA), followed by incubation with quenching buffer (192 mM glycine, $25 \mathrm{mM}$ Tris in PBS, pH 8.0) for $10 \mathrm{~min}$. Cells were detached by using ice-cold PBS buffer followed by centrifugation for $10 \mathrm{~min}$ at $1.000 \mathrm{~g}$. Cell lysis was performed with TBS (Trisbuffered saline) with $1 \%$ Triton-X100 and protease inhibitor mixture tablet (Roche Diagnostics, Mannheim, Germany) and centrifuged for $1 \mathrm{~min}$ at $13.000 \mathrm{~g}$. The supernatant (whole protein fraction) was incubated with $50 \mu \mathrm{l}$ of streptavidin-agarose beads (Pierce Biotechnologies, Rockford, IL, USA) for $2 \mathrm{~h}$ at $4^{\circ} \mathrm{C}$ while rotating. After removing the supernatant, beads were washed three times in TBS buffer. Biotinylated proteins were eluted by boiling with $50 \mu \mathrm{l}$ of $2 \mathrm{x}$ SDS buffer for $5 \mathrm{~min}$ at $95^{\circ} \mathrm{C} .40 \mu \mathrm{g}$ of surface proteins were analyzed by Western blot.

\section{Protein Degradation Analysis on Whole Cell Lysates}

HEK293 cells transiently expressing GlyR $\alpha 1$ wt or $\alpha 1$ variants were incubated under standard growth conditions with proteasome or lysosome inhibitors for specific time periods (all inhibitors have been obtained from Sigma-Aldrich, St. Louis, MO, USA). Cells were incubated with $1 \mu \mathrm{M}$ of MG132 for 1 , 2 , and $4 \mathrm{~h}$. The lysosome inhibitor leupeptin $(200 \mu \mathrm{g} / \mathrm{mL})$ was incubated for 6,12 , and $24 \mathrm{~h}$ together with the transfected cells.

At each time point, media was aspirated and cells were washed twice with PBS followed by incubation with CytoBuster Protein Extraction Reagent (Merck Millipore, Darmstadt, Germany) supplemented with protease inhibitor for $5 \mathrm{~min}$ at room temperature. The resulting cell suspension was centrifuged for $5 \mathrm{~min}$ at $16.000 \mathrm{~g}$ and $4^{\circ} \mathrm{C}$ resulting in a supernatant containing solubilized proteins.

Western Blot Analysis-For SDS-PAGE, 11\% polyacrylamide gels were freshly prepared, followed by Western blot on nitrocellulose membranes (GE Healthcare, Freiburg, Germany). Membranes were blocked for $1 \mathrm{~h}$ with 5\% BSA in TBS-T (TBS with $1 \%$ Tween 20). Primary antibodies were incubated overnight at $4{ }^{\circ} \mathrm{C}$. GlyR $\alpha 1$ wt and GlyR mutants were detected with the pan- $\alpha$ antibody MAb4a (Synaptic Systems, Göttingen, Germany), recognizing an epitope in the $\mathrm{N}$-terminus of the GlyRs (residues 96-105). In biotinylation experiments, cadherin was chosen as loading control detected by the pan-Cadherin antibody (Cell Signaling Technology, Danvers, MA, USA). For lysate analysis, GAPDH served as a loading control (Calbiochem, Darmstadt, Germany). Signals were detected using the ECLplus system (GE Healthcare, Freiburg, Germany).

\section{Immunocytochemical Staining}

Surface receptors were labeled following a co-transfection of HEK293 cells with GlyR $\alpha 1$ (wt) or $\alpha 1$ variants together with pDsRed-Monomer-Mem. pDsRed-Monomer-Mem (Clontech, Mountain View, CA, USA) encodes a fusion protein consisting of neuromodulin (GAP-43) and a red fluorescent protein used as plasma membrane marker. For surface staining of GlyR $\alpha 1$ in non-permeabilized cells, live cell staining was performed using MAb2b for $1 \mathrm{~h}$ at $4^{\circ} \mathrm{C}$ (1:500 in medium) as primary antibody recognizing a native epitope (residue 110 of mature protein at GlyR $\alpha 1$; Synaptic Systems, Göttingen Germany). Intracellular GlyR $\alpha 1$ staining was done following a co-transfection of HEK293 cells of $\alpha 1$ wt or $\alpha 1$ variants and pDsRed-ER. pDsRed-ER (Clontech, Mountain View, CA, USA) harbors the sequence information for a fusion protein of calreticulin and red fluorescent protein and can therefore be used as an ER marker. Cell staining was performed following fixation with $4 \%$ paraformaldehyde and $4 \%$ sucrose and a permabilisation/blocking step using PBS, $5 \%$ goat serum and $0.1 \%$ Triton-X100. Here, the primary antibody MAb4a (Synaptic Systems, Göttingen Germany) diluted 1:500 in PBS, $5 \%$ goat serum was used able to recognize the denatured epitope. Cells were washed three times with PBS before the secondary antibodies were applied for $30 \mathrm{~min}$. Here, goat antimouse Alexa488 or goat anti-mouse Cy3 antibodies (Dianova, Hamburg, Germany) were used 1:500 in PBS, 5\% goat serum. 
After a final wash, cells were embedded in Mowiol containing DAPI (1:20.000) to mark the nucleus.

\section{Compartmental Staining}

COS7 cells transiently expressing GlyR $\alpha 1$ (wt) or GlyR $\alpha 1$ variants were stained in permeabilized cells with primary antibodies MAb4a (Synaptic Systems, Göttingen Germany) and a polyclonal anti-calnexin antibody (1:500, Abcam, Cambridge, UK) for ER staining. The detection of ERGIC was done by the monoclonal ERGIC53 antibody (1:500, Enzo Life Science, Lörrach, Germany) and cis-Golgi stainings using a monoclonal antibody anti-GM130 (1:500, BD Transduction Laboratories, Heidelberg, Germany) together with a GlyR $\alpha 1$ specific antibody (Chemicon, Darmstadt, Germany). Secondary antibodies used were goat anti-mouse Cy3/Alexa488 and, goat anti-rabbit Cy3 (Dianova, Hamburg, Germany) diluted 1:500. All stainings were subjected to confocal microscopy on a DMIRE2 confocal microscope.

\section{Electrophysiology}

Maximal current amplifications $\left(I_{\max }\right.$ ) were measured by the patch clamp technique in a whole-cell configuration mode. Current signals were amplified with an EPC-9 amplifier (HEKA, Lambrecht, Germany). $24 \mathrm{~h}$ after transfection, whole cell recordings from HEK293 cells were performed by application of ligand (glycine) in different concentrations using a U-tube system. The extracellular buffer consisted of $137 \mathrm{mM} \mathrm{NaCl}$, $5.4 \mathrm{mM} \mathrm{KCl}, 1.8 \mathrm{mM} \mathrm{CaCl}_{2}, 1 \mathrm{mM} \mathrm{MgCl}, 11 \mathrm{mM}$ EGTA, $10 \mathrm{mM}$ HEPES, with a $\mathrm{pH}$ adjusted to 7.4 with $\mathrm{NaOH}$. The internal buffer was $120 \mathrm{mM} \mathrm{CsCl}, 20 \mathrm{mM} \mathrm{N}(\mathrm{Et})_{4} \mathrm{Cl}, 1 \mathrm{mM} \mathrm{CaCl}, 3 \mathrm{mM}$ $\mathrm{MgCl}_{2}, 11 \mathrm{mM}$ EGTA, $10 \mathrm{mM}$ HEPES with a $\mathrm{pH}$ adjusted to 7.4 with $\mathrm{CsOH}$. Recording pipettes were fabricated from borosilicate capillaries with an open resistance of about $4 \mathrm{M} \Omega$. Current responses were measured at a holding potential of $-60 \mathrm{mV}$. All experiments were carried out at room temperature. Error bars refer to S.E.M. values.

\section{Computational Methods}

The homology model of the GlyR $\alpha 1$ was generated by using the crystal structure of the glutamate-gated chloride channel (GluCl) at $3.35 \AA$ resolution (pdb code: $3 \mathrm{RIF}$ ) as a template (Hibbs and Gouaux, 2011). Both sequences were aligned according to the ClustalW algorithm using the default settings of the input form found at http://www.ch.embnet.org/software/ ClustalW.html (Thompson et al., 1994). The template structure was modified by removing the Fab molecule bound to $\mathrm{GluCl}$. Molecular modeling was performed using MODELLER9.9 (Sánchez and Sali, 2000) with the ligand module. The disulfide bridges were checked in the resulting model. The modeled structure was improved by 200 steps of conjugated gradient energy minimization using the Powell algorithm in Sybyl7.3 2002 St. Louis, MO, USA. The quality of the model was verified by WHAT_CHECK (Hooft et al., 1996). Mutations were introduced and positioned using Coot (Emsley et al., 2010). The visualization of the structures was performed with the PyMOL Molecular Graphics System, Version 1.7.6 (Schrödinger, LLC).

\section{Statistical Analysis}

Concentration-response curves were constructed from the peak current amplitudes obtained with at least seven appropriately spaced concentrations in the range 1-10.000 $\mu \mathrm{M}$ glycine. Using a non-linear algorithm (Microcal Origin), concentration-response data were first analyzed using the following Hill equation

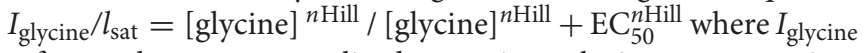
refers to the current amplitude at a given glycine concentration, $I_{\text {sat }}$ is the current amplitude at saturating concentrations of glycine, $\mathrm{EC}_{50}$ is the glycine concentration producing halfmaximal current responses, and $n_{\text {Hill }}$ is the Hill coefficient.

\section{RESULTS}

\section{Localization of Loop B Mutants within the GlyR}

A homology model of the GlyR $\alpha 1$ was generated from the $\mathrm{GluCl}$ crystal structure. The region between residues 157 and 162 is conserved within all GlyR subunits (Figure 1A). A recently discovered patient suffering from hyperekplexia carries a mutation at position G160 (pink) where glycine was mutated to arginine (G160R). Another patient mutation is the conversion of T162 (green) to methionine (T162M) again present in loop $B$ of the GlyR a1 ECD. Apart from these human mutations, we generated constructs harboring other amino acids at both positions G160 and T162 corresponding to residues in other Cys loop receptors at the appropriate loop B position (Figures 1A,B). G160A represents the change of a small hydrophobic side chain compared to glycine whereas G160S harbors a hydrophilic side chain. We also created T162A (small hydrophobic side chain), T162D (negatively charged side chain), T162N (hydrophilic side chain), and T162P (sterically demanding side chain). Both residues G160 and T162 are localized in the center of the neurotransmitter-binding site between adjacent subunits and close to the aromatic residue F159, which was suggested to directly interact with the incoming ligand glycine (Figure 1C).

\section{Impact of Residues 160 and 162 in Loop B on Receptor Expression}

Live cell stainings for surface expression showed no obvious differences between GlyR $\alpha 1$ wt and G160 variants (Figure 2A). GAP-43 expressed as a fusion protein with dsRed encoded on a co-transfected plasmid was used as membrane marker and for control of transfection efficiency (Figures 2A,B, 3). The plasma membrane detection of T162 variants at the single cell level did not consider differences to wt (Figure 2C). Although, $\alpha 1$ labeled cells were rare for T162M and T162A (Figure 3). An analysis of crude protein lysates (Figure 4A) was followed by protein quantification from Western blots after pull-down of biotinylated surface proteins by streptavidin binding. Protein lysates provided first evidences for differences in expression levels of $\alpha 1$ wt compared to $\alpha 1$ variants. The biotinylation method does allow a direct comparison between whole cell protein expression and surface protein expression. Here, significant differences of surface protein levels were observed for T162 variants compared to wt $\alpha 1$ (Figures 4B,C). The relative expression of the wt $\alpha 1$ 
A

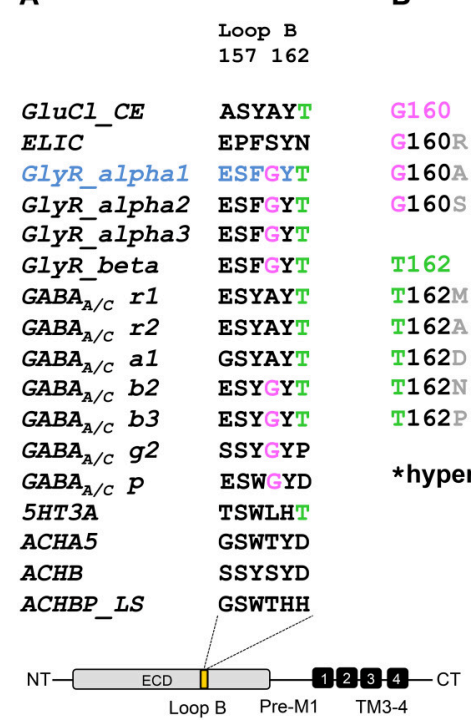

C Loop B
157162

ESF $G Y T$

ESFRYR*

ESFAYT

ESESYT

ESFGYT

ESFGYM*

ESFGYA

ESFGYD

ESFGYN

ESFGY

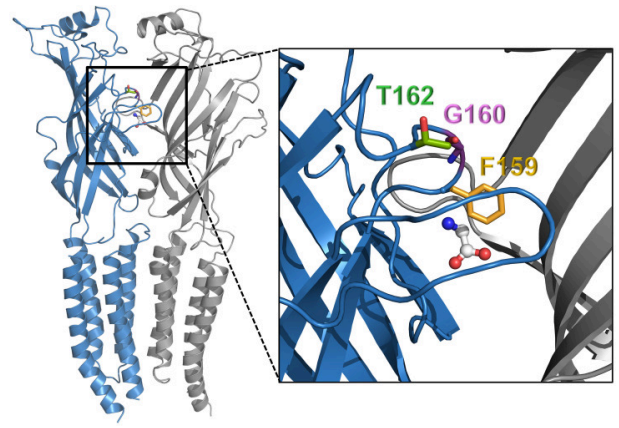

hyperekplexia mutation

FIGURE 1 | Overview domain architecture of the GlyR. (A) The loop B region of amino acid residues 157-162 is highly conserved within the family of Cys-Loop receptors. All glycine receptor subunits ( $\alpha 1$-blue, $\alpha 2, \alpha 3, \beta)$ carry the sequence of ESFGYT. A glycine is localized at position 160 (pink) and a threonine at position 162 (green). (B) Loop B mutations (gray letter) are shown and residues linked to hyperekplexia are marked by an asterisk (*). Glycine 160 was mutated into an alanine G160A and a serine G160S. Threonine at position 162 was mutated into alanine, aspartate, asparagine and proline (T162A, D, N, and P). (C) Representation of two GlyR $\alpha 1$ subunits of a receptor (blue and gray chain, respectively). For this homology model the crystal structure of GluCl (3RIF) was used as a template. Within the loop B region of amino acid 157-162 (enlarged inlet) between $\beta$-sheets 7 and 8 of the ECD residues G160 (pink), T162 (green), and F159 (yellow) are marked.

was set to $1(=100 \%)$. G160 variants were rather unaffected and showed whole cell as well as surface levels comparable to wt. T162M exhibited only marginal affected whole cell expression. All other T162 receptors did not differ in the overall expression levels (Figure 4C). T162M, T162D, T162P, and T162A revealed reduced protein levels at the cellular surface. T162N was the only mutant not affected in trafficking to the cell surface. The mutation T162M identified in a patient with hyperekplexia and T162D demonstrated significantly reduced surface expression levels (T162M $29 \pm 10 \%$, T162D $40 \pm 16 \%$; Figure 4C). Trafficking of T162P and T162A was decreased but did not reach significance (T162P $64 \pm 8 \%$, T162A $82 \pm 34 \%$ ). A determined portion of mutant GlyR protein seems to get stuck on its way to the cell surface most probably in the ER compartment as it has been shown previously for other recessive GlyR $\alpha 1$ mutants (Schaefer et al., 2015). The surface membrane fractions were not contaminated with cytosolic proteins (Figure 4D). Due to centrifugation of all cellular membranes followed by solubilization of proteins out of membranes, all fractions contained histone H3 usually forming tight associations with scaffold proteins of the inner nuclear membrane and are naturally postranslationally biotinylated (Figure 4D; Polioudaki et al., 2001; Bailey et al., 2008).

\section{Effect of the Human Mutation T162M on Protein Trafficking and Degradation}

T162M colocalizes with calreticulin, a chaperone present in the ER and important for protein quality control (Figure 5A). It has been shown for the nicotinic acetylcholine receptor, that calreticulin together with calnexin interact with newly synthesized glycoproteins in the ER, stabilizing the receptors and assisting in the folding process (Wanamaker and Green, 2007). Misfolded or unassembled protein is degraded via the ER-associated degradation (ERAD) pathways, e.g., via the proteasome. To determine ERAD of mutated GlyR $\alpha 1$, we blocked the proteasomal degradation with the proteasome inhibitor MG132 for different time periods (1, 2, and 4 h). We expected to observe protein accumulation since misfolded receptor proteins would normally be degraded via the proteasomal pathway. After a $1 \mathrm{~h}$ treatment with MG132, protein distribution of GlyR $\alpha 1$ wt and T162M was similar in the cytoplasm of transfected cells (Figures 5B,C). The protein expression pattern of wt was rather stable up to $2 \mathrm{~h}$ of treatment with only slight densities close to the nucleus at $4 \mathrm{~h}$ presence of the blocking agent (Figure 5B). In contrast, accumulation of T162M was already present after $2 \mathrm{~h}$ and even more prominent after $4 \mathrm{~h}$ of treatment (Figure 5C). MG132 is a highly potent, cell-permeable proteasome inhibitor. Since long incubation with MG132 is toxic to cells, we quantified the GlyR $\alpha 1$ wt and T162M protein amounts following proteasomal blocking following a $2 \mathrm{~h}$ presence of MG132 demonstrating a significant increase of T162M protein compared to wt protein (Figures 6A,B). Following $1 \mathrm{~h}$ incubation with MG132, the T162M starts to accumulate, which is more prominent at $2 \mathrm{~h} \mathrm{MG132}$ treatment. The lower degradation bands do also increase in intensity between 1 and $2 \mathrm{~h}$ presence of the blocking agent (Figure 6A). This might argue for less stable protein and higher turnover rates of the mutated GlyRs. The GlyR $\alpha 1$ wt protein is not 


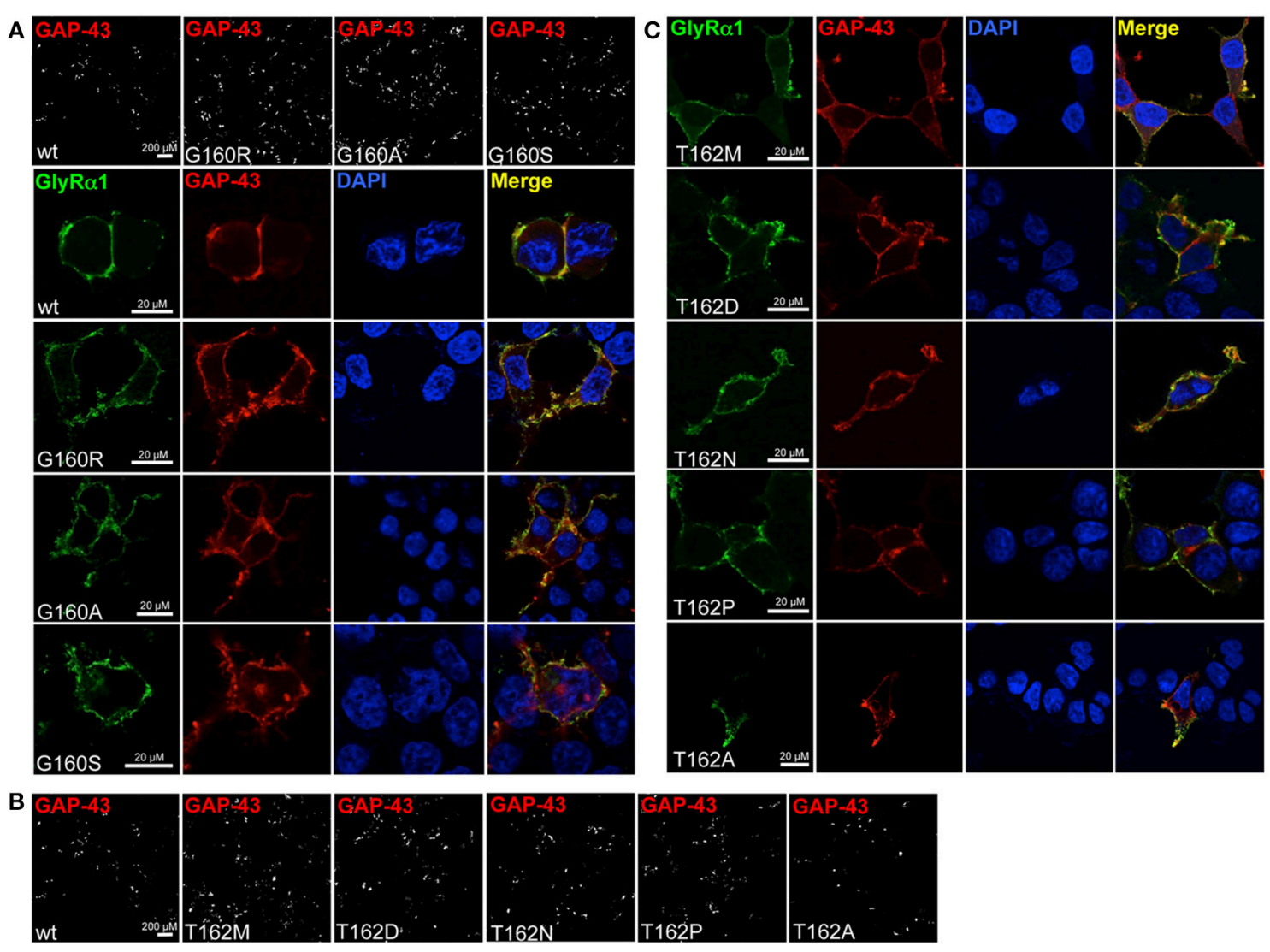

FIGURE 2 | Integration of GlyR variants into the cellular membrane. Different $\alpha 1$ mutants were expressed in HEK293 cells following co-transfection of a marker for membrane expression (GAP-43 coupled to dsRed). (A) Overview images (first line) represent controls for transfection efficiency (GAP-43) for wt and G160 variants. Below GlyR $\alpha 1$ homomers (green) expressed at cell surface (GAP-43 marker) are shown. G160R, G160A, and G160S mutants are well integrated into the cell surface. (B) Transfection efficiency controls for cotransfections of T162 mutants together with GAP-43 coupled to dsRed. (C) Mutant GlyRs at position 162 (T162M, T162N, T162D, T162P, and T162A) stained at the cell surface. All GlyR $\alpha 1$ were detected with the monoclonal MAb2b antibody. DAPI was used for staining of the nuclei.

affected at its protein level by a $2 \mathrm{~h}$ treatment with MG132. It has been shown that ubiquitinated GlyRs expressed at the cell surface are degraded by subsequent internalization and lysosomal pathways (Büttner et al., 2001). To test if the mutant T162M also uses this pathway for degradation, leupeptin was utilized to block lysosomal degradation. Following treatment with leupeptin, the T162M mutation did not show any differences upon leupeptin treatment after $6 \mathrm{~h}$ but reduced protein levels after a $12 \mathrm{~h}$ treatment with leupeptin in contrast to GlyR $\alpha 1 \mathrm{wt}$ (Figure 6A). The mutant T162M is still degraded in the presence of leupeptin arguing for ongoing degradation using pathways independent of degradation via lysosomes compared to $\alpha 1 \mathrm{wt}$.

Subcompartmental analysis of GlyR $\alpha 1$ wt and T162M was performed for the compartments ER, ERGIC and cisGolgi in Cos7 cells using co-stainings with marker proteins present in these compartments. Cos-7 cells have been used for subcompartmental analysis due to their large cytoplasm in comparison to HEK293 cells. Calreticulin, a chaperone localized at the ER exit sites for folded proteins, co-localized with the T162M but only marginally with GlyR $\alpha 1$ wt (Figure 7A). The GlyR $\alpha 1$ wt instead is observed at the outer most cytoplasm and the membrane. T162M forms large accumulations in the ER. ERGIC-53 is a protein of the ER-Golgi intermediate compartment. Subpopulations of T162M pass the ERGIC and cycle toward the cis-Golgi compartment where co-localisation was observed with GM130 (Figures 7B,C). The GlyR $\alpha 1$ wt was only present at some locations in ERGIC but no wt protein was detectable in cis-Golgi, arguing for a fast forward trafficking of the GlyR $\alpha 1$ wt receptors. The large ER accumulations of mutated T162M protein in the ER might not only result from the recognition of abnormally folded or incompletely assembled $\mathrm{T} 162 \mathrm{M}$ protein. Retrograde transport mechanisms from cisGolgi and ERGIC might also underlie this phenomenon.

\section{Changes in Ligand Potencies of GlyR $\alpha 1$ Loop B Variants}

The physiological characterization of the mutant receptor populations was done by electrophysiological measurements using whole-cell recordings following expression in HEK293 cells. In HEK293 cells, the $\mathrm{EC}_{50}$ of glycine for GlyR $\alpha 1$ wt has been reported between 15 and $60 \mu \mathrm{M}$. Therefore, we used a glycine concentration of $100 \mu \mathrm{M}$ to determine whole-cell 


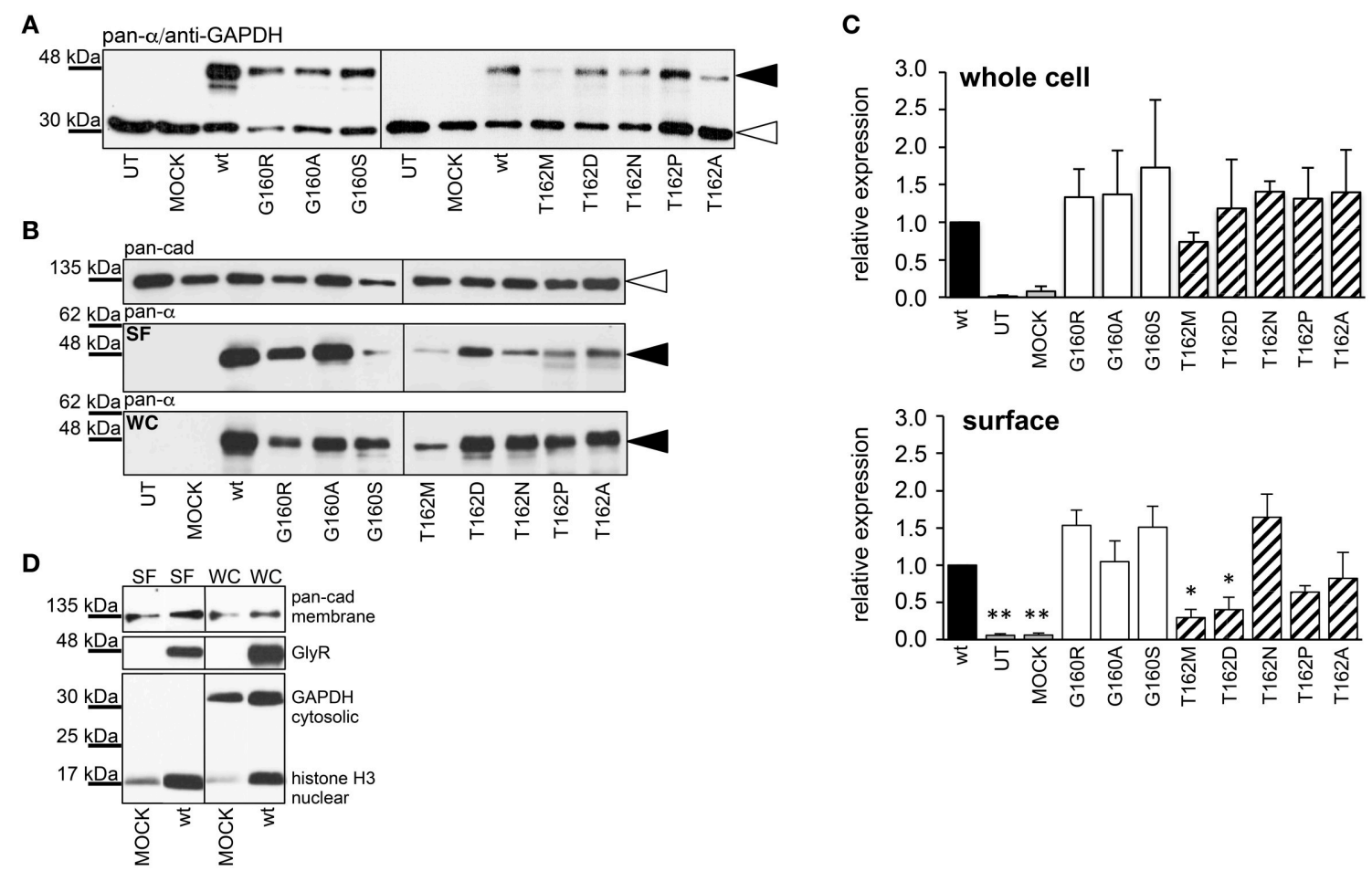

FIGURE 4 | Quantitative expression levels of mutant GlyRs. (A) Crude HEK293 whole cell lysates transiently expressing GlyR $\alpha 1$ (wt) or $\alpha 1$ variants. Loading control GAPDH $37 \mathrm{kDa}$. (B) Biotinylation pull down experiments to quantify the protein expression of GlyR $\alpha 1$ variants and to distinguish between whole cell (WC) and surface (SF) protein levels. The obtained data were taken from 4 independent experiments. The overall expression seems to be unaffected. Note, differences between surface expression levels of wt and G160 compared to T162 variants. Pan-cadherin was used as a loading control. The GlyR $\alpha 1$ variants were stained at the appropriate molecular weight of $48 \mathrm{kDa}$ (black arrowheads), pan-cadherin appeared at $135 \mathrm{kDa}$ (white arrowheads). (C) Quantification of protein levels determined from whole cell (WC) and surface (SF) fractions from 4 independent experiments normalized to pan-cadherin (membrane marker protein). The expression levels are shown in comparison to wild type ( $w t=1)$. Error bars refer to standard error of the mean S.E.M. values. ${ }^{*} p<0.05,{ }^{* *} p<0.01$ were considered significant. (D) Control for purity of surface protein fraction. Surface (SF) and whole cell (WC) fractions were loaded from MOCK and wt $\alpha 1$ transfected HEK293 cells. Both fractions were stained for the cytosolic protein GAPDH ( $37 \mathrm{kDa}$ ), the membrane marker pan-cadherin (135 kDa) and the nuclear marker histone H3 (17 kDa). Note, histones were stained in all fractions due to (i) their localization attached to the inner nuclear membrane, (ii) naturally occurring posttranslational biotinylation of histones, and (iii) binding to streptavidin beads following spin-down of membranes and solubilization of membrane attached proteins.

currents resulting in maximal currents for the wt expressing cells. $I_{\text {rel }}$ currents of G160 mutants (white bars, Figure 8A) were similar to wt. G160R evoked $69 \pm 10 \%$ of the wt current, G160A evoked $92 \pm 12 \%$ of wt and G160S ended up with $68 \pm 22 \%$ of wt current. The reduction of the observed inward currents for G160 mutant was, however, not significant (Figure 8A, Table 1). T162 $\alpha 1$ variants (gray striped bars, Figure 8A) showed significantly decreased inward currents at $100 \mu \mathrm{M}$ glycine. T162M could only achieve $11 \pm 5 \%$ of $\mathrm{I}_{100 \mu \mathrm{M}}$ compared to wt. T162N achieved $13 \pm 3 \%$ and T162P $29 \pm 10 \%$. T162A was able to reach $4 \pm 1 \%$ of maximum current of wt (Figure 8A). Except T162D, all variants were able to form functional receptors but evoked less inward currents. A reduction of the inward current might be due to the observed low expression of these mutated receptor complexes at the cellular surface. The reduction of surface expression might not be the major reason for inward current reduction as demonstrated by $\mathrm{T} 162 \mathrm{~N}$, which is indistinguishable from wt in cell surface receptor numbers but resulted in a reduction of $85 \%$ of the wt response. For T162D less than 1\% of wt currents were measured in two out of five cells recorded arguing for a non-functional mutation.

Application of $100 \mu \mathrm{M}$ glycine did not result in obvious changes for G160 variants. In addition, trafficking of G160 mutants is not affected. Determination of glycine potency however showed significant differences. Using 7 different glycine concentrations in a range of $1-1.000 \mu \mathrm{M}$ glycine, all G160 mutants showed reduced ligand potencies $\left(\mathrm{EC}_{50}\right)$. GlyR $\alpha 1$ wt achieved its half maximal current at a concentration of $36 \pm 10 \mu \mathrm{M}$. The $\mathrm{EC}_{50}$ value determined for $\mathrm{G} 160 \mathrm{R}$ was 92 $\pm 13 \mu \mathrm{M}$. A maximal concentration of glycine $(1 \mathrm{mM})$ was not sufficient to reach saturation for GlyR $\alpha$ variants G160A and G160S. However, both variants exhibit largely decreased agonist potencies with $\mathrm{EC}_{50}$ values $\geq 383 \mu \mathrm{M}$ for $\mathrm{G} 160 \mathrm{~A}$ and $\geq 184 \mu \mathrm{M}$ for G160S (Figure 8B). The glycine concentration to activate $50 \%$ of maximal currents $\left(\mathrm{I}_{\max }\right.$ ) was increased by a factor of 3-10 for G160 mutants. The effect was even more prominent for $\mathrm{T} 162 \mathrm{M}$ with more than a 21 fold increase in glycine $\mathrm{EC}_{50}$. The $\mathrm{EC}_{50}$ for the mutant $\mathrm{T} 162 \mathrm{M}$ was $776 \pm 96 \mu \mathrm{M}$ (Figure 8B). 
A
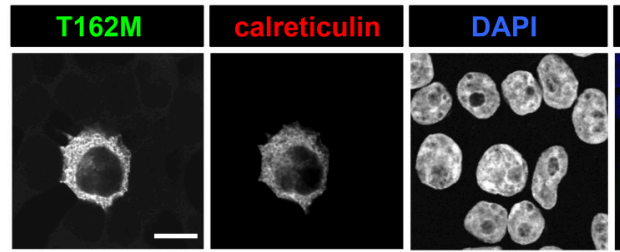

MERGE

B GlyRa1

DAPI

MERGE
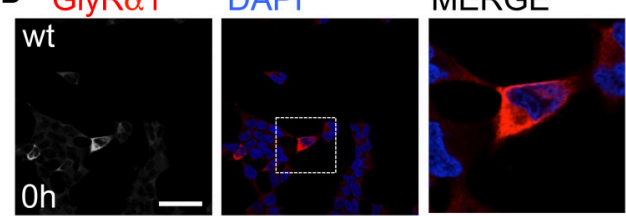

\section{GlvRa1}

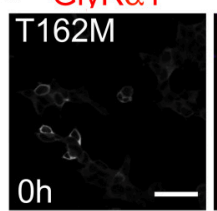

DAPI
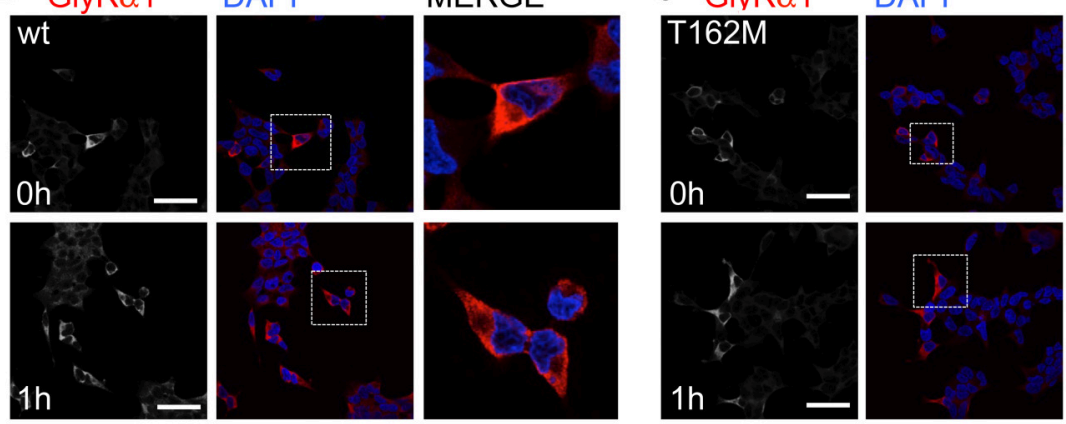

MERGE
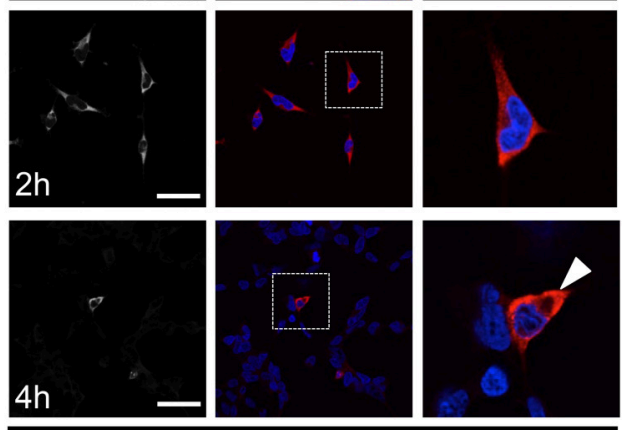

MG132
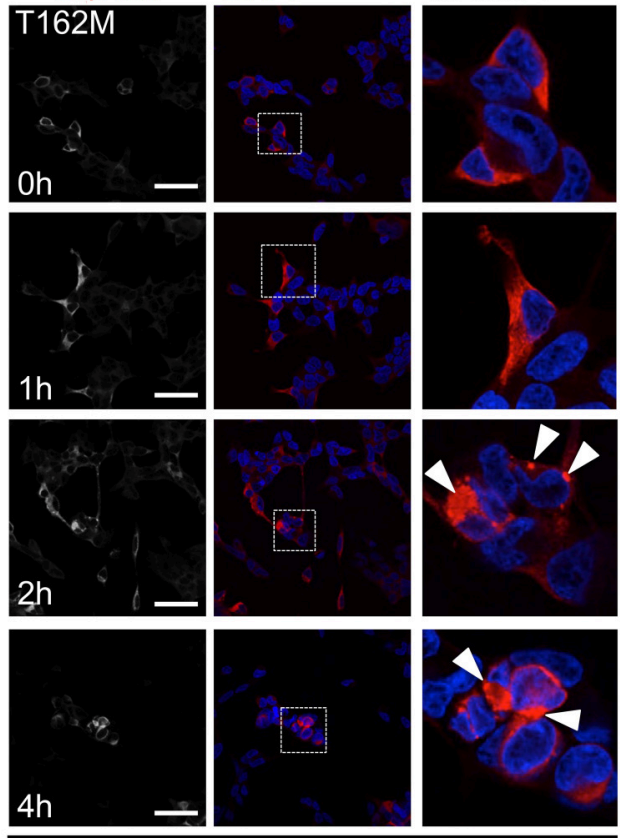

MG132

FIGURE 5 | ER accumulation of T162M. (A) Following cotransfection into HEK293 cells, intracellular stainings of GlyR $\alpha 1$ T162M (MAb4a, green) and the ER marker calreticulin expressed as a fusion protein coupled to dsRed (red). (B) Inhibition of the proteasomal pathway using MG132 for 1,2 , and $4 \mathrm{~h}$. Following $2 \mathrm{~h}$ presence of MG132, wt transfected cells (MAb4a, red signal) seemed rather unaffected in protein distribution. (C) In contrast, T162M (MAb4a, red) showed large accumulations in the cellular lumen (white arrowheads, third lane, right picture). Lower lane represents pictures after $4 \mathrm{~h}$ treatment with MG132. Enlarged images are shown in right column. GlyR wt (B) signal is dense, whereas T162M (C) is characterized by large protein aggregations localized close to the nucleus.

\section{Conformational Changes by an Introduction of a Negatively Charged Residue in Loop B}

Since the mutant T162D did not result in functional ion channels although present to some extent at the cellular surface, we followed T162D trafficking in transfected Cos7 cells (Figure 9A). T162D passed the ERGIC and the cis-Golgi with no obvious changes compared to T162M (Figure 7A). Similar to T162M, ER accumulation was observed. The position T162 in the GlyR seems to influence protein folding followed by ER export (Figure 9A). When high saturating concentrations of glycine $(10 \mathrm{mM})$ were applied to cells expressing T162D, the receptors responded with maximal currents indistinguishable from GlyR $\alpha 1$ wt (Figure 9B). Hence, the mutant T162D did not result in non-functional ion channels rather physiological concentrations of glycine are not sufficient to activate these GlyR channels. Interestingly, the $\mathrm{T} 162 \mathrm{D}$ channels close faster during ligand washout compared to wt channels (Figure 9C). Homology modeling of the T162D mutation revealed that the introduction of a negatively charged aspartate likely allows a salt bridge formation with the positively charged arginine R119 present at the neighboring GlyR subunit in the pentameric receptor complex (Figure 9D). Thus, a primary change, putatively in the fold and flexibility of the ligand-binding site due to saltbridge formation, less ER export and integration in the outer cellular membrane resulted secondary in an ion channel with an unfavorable conformation of the ligand-binding pocket.

\section{DISCUSSION}

Glycinergic disinhibition results from either disrupting the functionality of glycine receptors or trafficking disabilities. So far, mutations in the gene GLRA1, the major common cause for the neuromotor disorder hyperkplexia associated with disturbances in glycinergic neurotransmission, have been categorized into dominant mutations associated with functional 


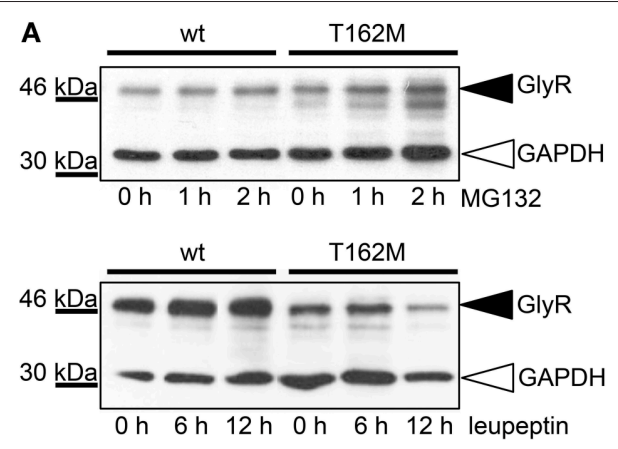

B

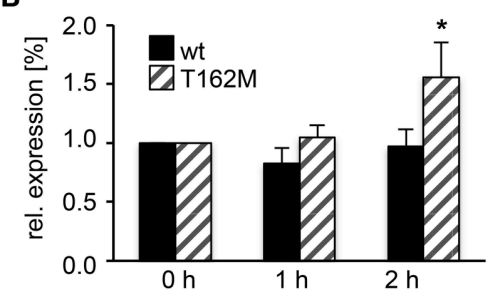

FIGURE 6 | T162M degradation via the proteasomal degradation pathway. (A) MG132 was added to transfected HEK293 cells expressing either wt or T162M for 0, 1, and $2 \mathrm{~h}$. Following incubation with the proteasomal blocker, cell lysates ( $40 \mu \mathrm{g}$ per lane) were analyzed by Western blotting (upper blot) using the GlyR antibody MAb4a (black arrowhead, $48 \mathrm{kDa}$ ). GAPDH $37 \mathrm{kDa}$ served as a loading control (white arrowhead). Inhibition of cellular lysosomes by leupeptin for 0,6 , and $12 \mathrm{~h}$ (lower blot), GAPDH 37 kDa. GlyR protein is marked by black arrowhead. (B) Quantification of protein levels after MG132 incubation for 1 and $2 \mathrm{~h}$ from 4 independent experiments. T162M increased after $2 \mathrm{~h}$ proteasomal block, ${ }^{*} p<0.05$.

disruptions and recessive mutants affecting receptor biogenesis (Chung et al., 2010; Bode and Lynch, 2014). Recently, we could demonstrate that disrupted trafficking does not necessarily result in ER accumulation and degradation. Rather, subpopulations of receptor are able to circumvent the ER quality control and traffic through the secretory pathways of the Golgi compartment. However only a minority is able to integrate into the outer membrane. The receptor numbers at the cell surface are insufficient to enable a normal glycinergic function (Schaefer et al., 2015). Here, we analyzed in detail human mutations localized within the GlyR ligand-binding sites resulting from different modes of inheritance dominant-G160R and recessive-T162M.

To get deeper insights into the importance of residues within the ligand-binding site we mutated the affected residues into amino acids present in other subtypes of the Cys-loop receptor family - the closely related inhibitory receptors of the $\mathrm{GABA}_{\mathrm{A} / \mathrm{C}}$ family, the prokaryotic receptor ELIC and the C.elegans receptor GluCl harboring the highest sequence identity to the glycine receptor family among all Cys-loop receptor members (Hilf and Dutzler, 2009; Hibbs and Gouaux, 2011). The mutant G160R as well as the other two mutants G160A and G160S showed no differences in expression levels compared to the wt receptor $\alpha 1$. This was demonstrated by independent methods of immunocytochemical as well as proteinbiochemical analyses.
Originally, G160R was identified in a patient with a dominant mode of inheritance (Schaefer et al., 2015). G160 is localized in direct neighborhood to F159, which has been shown to be important for a cation- $\pi$ interaction with the incoming ligand glycine (Schmieden et al., 1993; Pless et al., 2008). In a recent study using a series of unnatural amino acids, a cation$\pi$ interaction between F159 and the amino groups of $\beta$-alanine and taurine was also determined. Compared to the interaction with glycine, the strength of interactions with taurine and $\beta$ alanine was significantly weaker. This might be due to a different orientation of the partial agonists within the ligand-binding pocket (Pless et al., 2011). Similarly, mutations of other aromatic residues contributing to the ligand-binding interface, e.g., F63, F207 glycine concentrations of up to $300 \mathrm{mM}$ failed to saturate the current responses (Grudzinska et al., 2005). Our analysis of the ligand-binding potencies revealed a 3-10-fold decrease in ligand-efficacy of glycine demonstrating the importance of the small G160 to enable the correct conformation of the ligand-binding pocket for the natural agonist. Interestingly, the positively charged side chain of the arginine did not result in a more drastically affected ligand potency compared to a small side chain change e.g., in G160A. We conclude that the smallest amino acid glycine harboring a hydrogen instead of a side chain at position 160 of the human GlyR $\alpha 1$ is essential to provide the optimal ligand-binding conformation for the neurotransmitter glycine. Nevertheless, we cannot exclude other factors such as differences in the transduction pathways following ligand-binding enabling channel opening.

Residue T162 is also localized within the binding site. The underlying mechanism of the human mutant T162M seems to be a combination of affected biogenesis and functional disruption. Only $50 \%$ of $\mathrm{T} 162 \mathrm{M}$ receptors are able to reach the cell surface obtained following transfection into heterologous expression systems. Although, ER accumulation was observed, the mutant was also detectable in compartments such as the ER-Golgi intermediate compartment ERGIC as well as the cisGolgi. Therefore, the overall protein fold of T162M and T162D, $\mathrm{P}$, and A does not lead to a misfold resulting in lack of ER release (Lippincott-Schwartz et al., 2000; Hebert and Molinari, 2007). The subpopulations of T162 mutants reaching the cell surface are, however, not sufficient to enable functionality of the GlyR complex. We hypothesize retrograde signaling of mutated $\alpha 1$ subunits initialized by so far unknown control proteins within the ERGIC and the cis-Golgi compartment resulting in proteasomal degradation (ERAD degradation). Proteasomal degradation has been shown for other recessive mutants (Villmann et al., 2009). Moreover, a higher turnover of mutated $\alpha 1$ receptor was exhibited by pulse-chase experiments. Here, we could demonstrate that the mutated $\mathrm{T} 162 \mathrm{M}$ resulted in cytoplasmic accumulation after a $2 \mathrm{~h}$ treatment with the proteasomal blocker MG132 in comparison to wt. For the wt receptor, accumulation was first observed after a $4 \mathrm{~h}$ treatment but with much less local protein close to the nuclear area compared to T162M. Such accumulations in aggrosome-like structures close to the nucleus have been demonstrated following proteasomal blocking. Aggrosomes assemble to process misfolded proteins that cannot well be handled by the ubiquitin-proteasome 
A
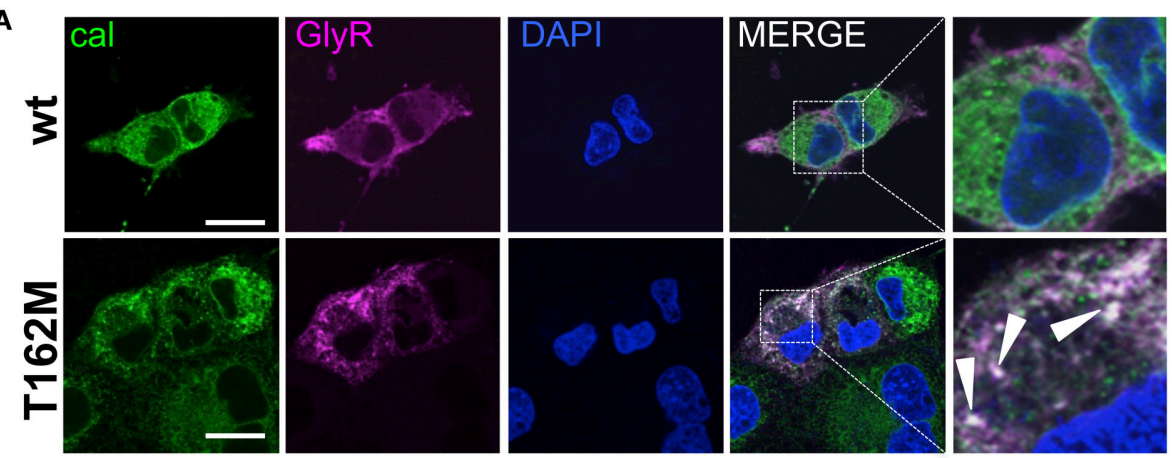

B
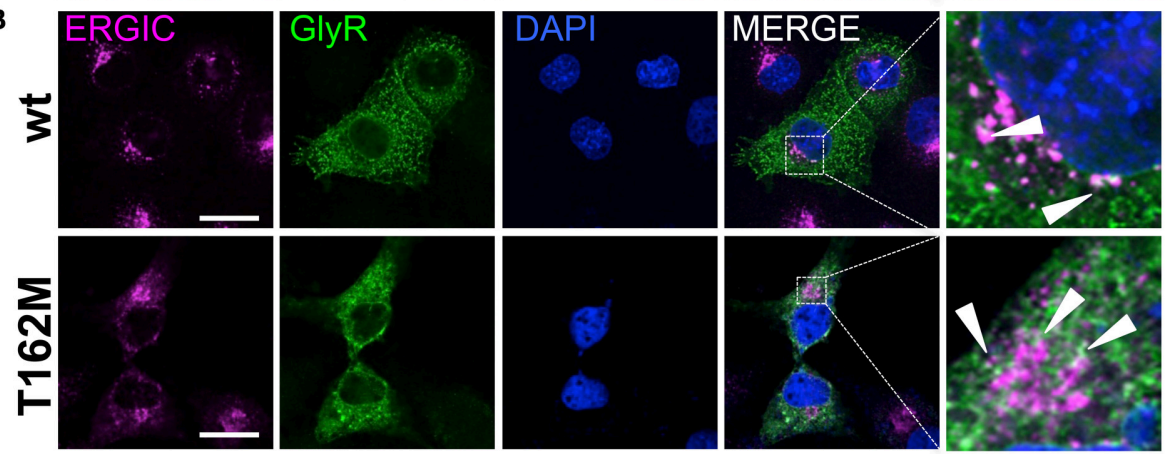

C
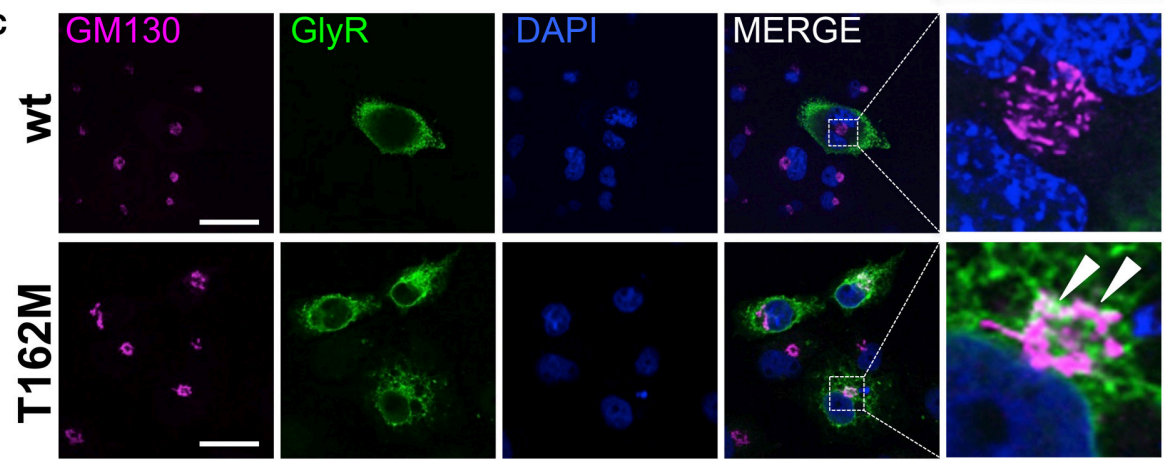

FIGURE 7 | Trafficking routes of T162M. (A) Costaining of T162M (MAb4a, red) and calreticulin (green), a chaperone and ER quality control protein in the ER in transfected Cos7 cells. Note again large T162 accumulations in the ER compared to wt (upper lane, white dots marked by white arrowheads). (B) ERGIC co-staining of GlyR wt and T162M (anti- $\alpha 1$, green) together with ERGIC-53 (pink). (C) Cis-Golgi staining using GM130 (pink) as a marker of the secretory Golgi compartment. Mutant GlyR T162M (anti- $\alpha 1$, green) was visible in the cis-Golgi compartment. The white bar represents $30 \mu \mathrm{m}$. Right column represents enlargements of merged images (white dotted boxes).

pathway (Goldberg, 2003). Our calculation of T162M protein following a block of proteasomal degradation resulted in a significant increase of T162M protein levels after $2 \mathrm{~h}$ presence of MG132.

Endocytosis followed by lysosomal degradation has been shown for membrane-associated GlyR $\alpha 1$ wt (Büttner et al., 2001). The incorporation of membrane proteins into endosomal membranes is a common pathway for membrane-bound receptor proteins. The GlyR $\alpha 1$ is a stable glycoprotein with a half-life of approximately 2 days (Hoch et al., 1989). Following a $12 \mathrm{~h}$ presence of the lysosomal blocker leupeptin, we indeed could demonstrate accumulations of wt GlyR $\alpha 1$. Therefore, lysosomal degradation does indeed represent the major degradation pathway for the GlyR $\alpha 1$ wt protein.
In contrast, the T162M mutant was observed at much lower receptor protein levels arguing for ongoing degradation in the presence of leupeptin using pathways other than the lysosomal pathway. However, we cannot exclude that the fraction of T162M cell surface receptors might use lysosomal pathways for degradation. The proportion of T162M receptors that does not traffick to the cell surface is degraded via the proteasomal pathway.

The fraction of T162 mutant receptor at the cell surface diminishes glycinergic function to only $20 \%$ of wt activity at a glycine concentration of 3 -fold above $\mathrm{EC}_{50}$ for wt $\alpha 1$. At glycine concentrations above physiological levels 3-10 mM the functionality of T162 mutants was indistinguishable from wt receptors. Concentrations of up to $3.5 \mathrm{mM}$ glycine can be 
A
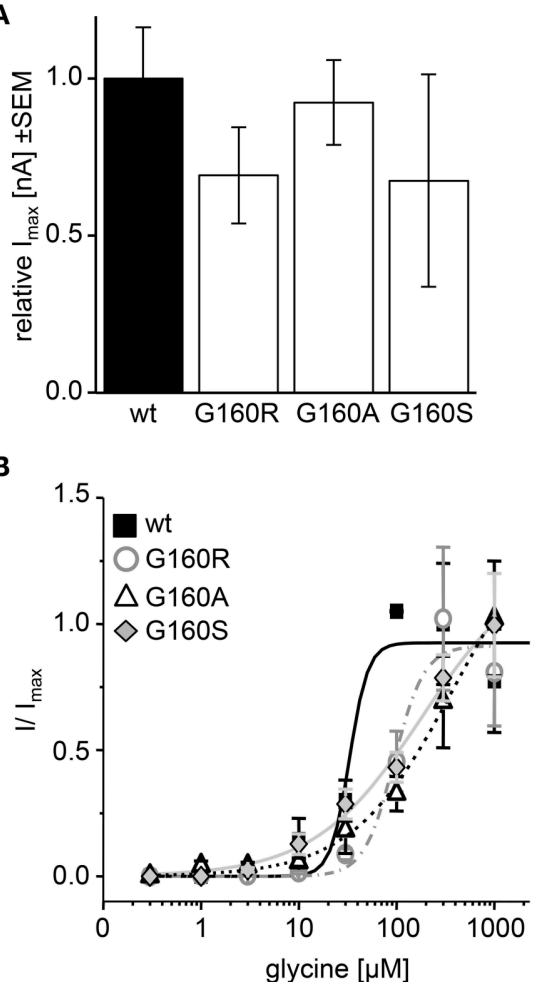
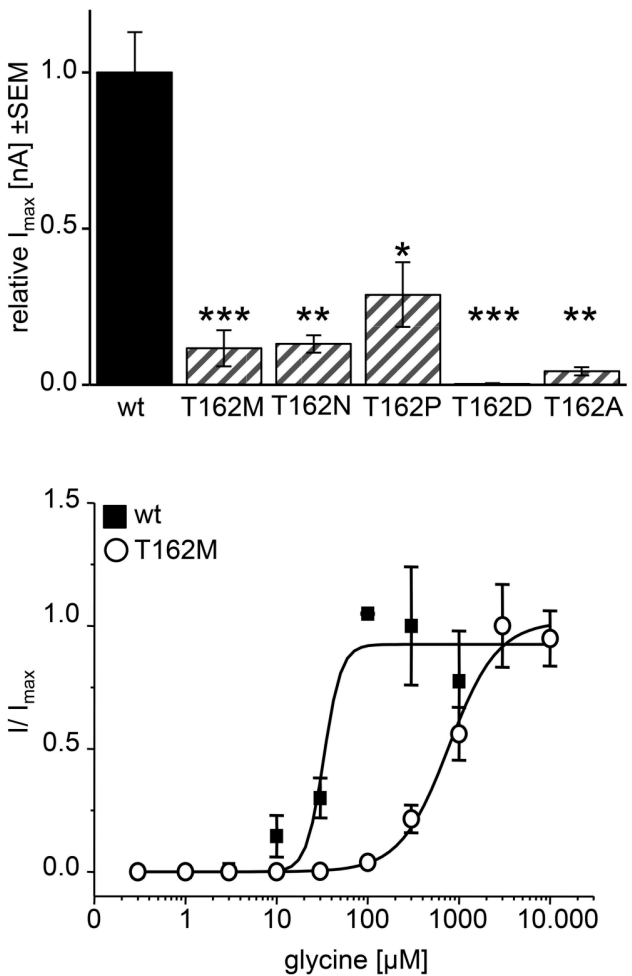

FIGURE 8 | Physiological changes in agonist potency due to affected loop B residues. Whole cell recordings on HEK293 cells transiently expressing GlyR a1 or mutant GlyR were performed to analyze receptor functionality and ligand potency. (A) Relative maximal currents (Imax) of G160 variants (left graph, white bars) determined at $100 \mu \mathrm{M}$ glycine. Note, all T162 variants show reduced maximal currents (right graph, gray striped bars). n, number of independent measurements; $n=7 ;{ }^{*} p<0.05,{ }^{* *} p<0.01,{ }^{* *} p<0.001$. (B) EC 50 measurements using glycine concentrations of 1, 3, 10, 30, 100, 300, 1000, 3000, and 10.000 $\mu \mathrm{M}$. GlyR $\alpha 1$ wt-black square, solid line; G160R-circles, dot-dashed line; G160A-triangle, dashed line; G160S-diamond, gray line; T162M-white circles, solid line. $1 \mathrm{mM}$ glycine was used as a standard glycine concentration to determine $1 / \operatorname{lmax} . n=5-6$.

TABLE 1 | Functional properties obtained from GlyR $\alpha 1$ mutants at positions 160 and 162.

\begin{tabular}{|c|c|c|c|c|c|c|c|}
\hline Clone & $\begin{array}{c}\text { Number of } \\
\text { independent experiments }\end{array}$ & $\begin{array}{l}\text { Expression at } \\
\text { cell surface }\end{array}$ & $\begin{array}{l}\text { Whole cell } \\
\text { expression }\end{array}$ & $\begin{array}{l}\text { Number of } \\
\text { cells }\end{array}$ & $I_{\max }[p A] \pm \mathrm{SEM}$ & $\begin{array}{l}\text { Number of } \\
\text { cells }\end{array}$ & $\mathrm{EC}_{50}$ gly $[\mu \mathrm{M}] \pm$ SEM \\
\hline GlyR $\alpha 1$ wt & 4 & +++ & +++ & 7 & $1571 \pm 260$ & 5 & $36 \pm 10$ \\
\hline MOCK & 4 & - & - & - & - & - & - \\
\hline$\alpha 1 \mathrm{G} 160 \mathrm{R}$ & 4 & ++ & +++ & 7 & $1089 \pm 167$ & 5 & $92 \pm 13$ \\
\hline GlyR $\alpha 1$ wt & 4 & +++ & +++ & 17 & $2916 \pm 371$ & 5 & $36 \pm 10$ \\
\hline a1 T162M & 4 & + & ++ & 10 & $309 \pm 154^{\star \star \star}$ & 6 & $776 \pm 96$ \\
\hline a1 T162A & 4 & ++ & +++ & 5 & $148 \pm 45^{\star \star}$ & - & n.d. \\
\hline a1 T162D & 4 & + & +++ & 7 & $10 \pm 6^{\star \star \star}$ & - & n.d. \\
\hline$\alpha 1 \mathrm{~T} 162 \mathrm{~N}$ & 4 & +++ & +++ & 5 & $447 \pm 96^{\star \star}$ & - & n.d. \\
\hline
\end{tabular}

*UT refers to untransfected cells and MOCK to GFP transfected cells used as controls. n, number of independent experiments; SEM (standard error of the mean), n.d. not determined, $p$-values refer to the appropriate $W T{ }^{*} p<0.05,{ }^{* *} p<0.01,{ }^{* \star} p<0.001$. 

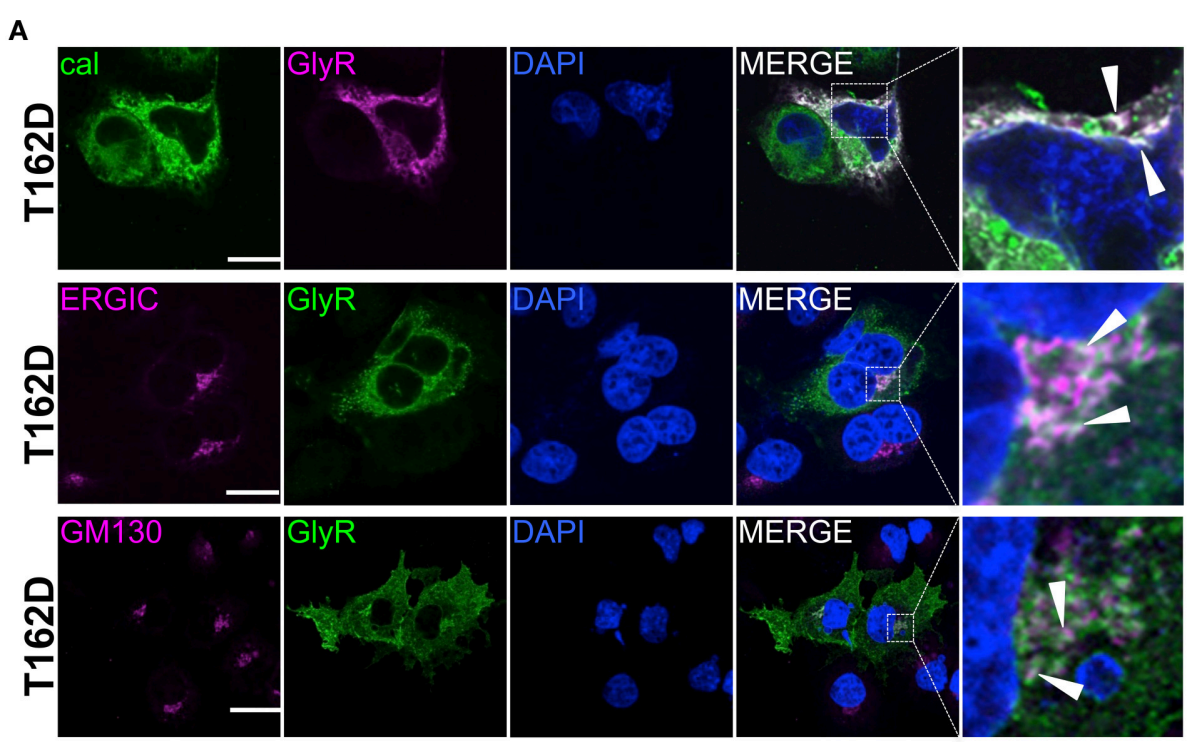

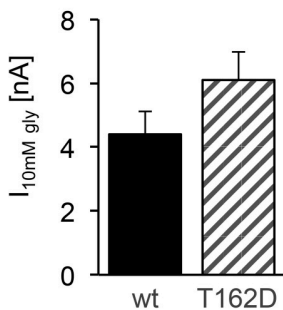

C

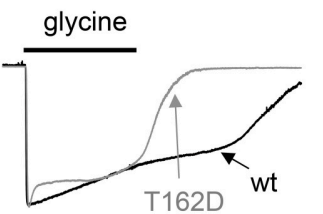

D

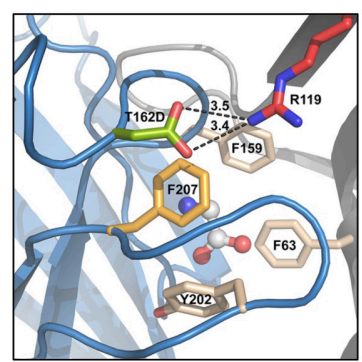

FIGURE 9 | Subcompartmental distribution of T162D. (A) Transfected Cos7 cells were used to co-stain GlyR a1 T162D (pink) with calreticulin (green). Large ER accumulations (white dots marked by white arrow heads) were obvious (right picture, first lane). Costaining of T162D (anti- $\alpha 1$, green) with ERGIC (pink, second lane), colocalization with GM130 (pink, third lane). Right pictures demonstrate enlarged areas of the white dotted box in the merged picture. (B) Whole cell maximal currents of T162D compared to $\alpha 1 \mathrm{wt}$ at saturating concentrations of glycine (10 mM). (C) Single traces of $10 \mathrm{mM}$ recordings from wt and T162D. Note, the fast channel closure compared to wt channels during washout of very high glycine concentrations. (D) View from the side onto the ligand-binding site at the interface of two adjacent GlyR subunits (blue and gray), with bound glycine (in ball-and-stick representation). Residue D162 is marked in green, F159 in beige, further aromatic residues lining the glycine binding site (Y202, F63) are beige and R119 shown in red with putative salt bridge indicated by black dashed lines and distances given in $\AA$. Note, F63 and R119 are localized at the neighboring subunit.

reached during activation (Beato, 2008), however, the fraction of cell surface receptors might still be too low to enable inhibitory neurotransmission in the human organism. The $\mathrm{EC}_{50}$ for $\mathrm{T} 162 \mathrm{M}$ shows a 21 -fold increase indicating a disruption of the ligand-binding site. The artificial mutant T162D resulted in non-functional channels following glycine application of concentrations 3 -fold above $\mathrm{EC}_{50}$ for wt $\alpha 1$. At very high concentrations of glycine, mutated $\mathrm{T} 162 \mathrm{D}$ receptors were able to open. A conformational change required to open the ion channel pore seems therefore not be hindered by the mutation rather high glycine concentrations are necessary to allow activation of channels.

Modeling of the mutant T162D demonstrated that the mutation of T162 into an aspartate might result in novel interactions with other residues from the neighboring subunit. The introduction of the negatively charged aspartate would enable the formation of a salt bridge together with R119 from the neighboring subunit. One might speculate that the novel salt bridge stabilizes the protein conformation and does not result in misfolding allowing receptor forward trafficking from the ER to further cell compartments. Following integration into the cell surface the receptor configuration is less favorable to get activated by the neurotransmitter glycine. In vivo, such GlyR $\alpha 1$ mutants may provide cell stress to motoneurons resulting in a more severe phenotype of these patients compared to patients carrying a NULL-allele (Brune et al., 1996; Becker et al., 2006) suffering from a rather mild form of hyperekplexia.

In summary, residues G160 and T162 localized in loop $B$ of the GlyR $\alpha 1$ protein lining the ligand-binding pocket are essential determinants for ligand potency. Both residues when mutated lead to significant decreases in ligand potency. In contrast to G160, where the functional disturbances are a primary consequence of the mutation, the observed functional impairment of T162 mutants is most probably a secondary effect. Primarily, lack of threonine 162 leads to changes in the overall protein biogenesis, but mutant receptors are still sufficient 
to circumvent the ER quality control. The small fraction of cell surface receptors is not able to sustain normal glycinergic neurotransmission. Hence, loop B residues are crucial elements within the conformation of the ligand-binding pocket.

\section{AUTHOR CONTRIBUTIONS}

Participated in Research design-CV. Conducted experimentsCV, SA, NS, GL, and DK. Performed data analysis-HM, CD, HS, $\mathrm{CV}, \mathrm{SA}, \mathrm{GL}, \mathrm{NS}$, and DK. Wrote the manuscript-CV, SA.

\section{REFERENCES}

Bailey, L. M., Ivanov, R. A., Wallace, J. C., and Polyak, S. W. (2008). Artifactual detection of biotin on histones by streptavidin. Anal. Biochem. 373, 71-77. doi: 10.1016/j.ab.2007.09.003

Beato, M. (2008). The time course of transmitter at glycinergic synapses onto motoneurons. J. Neurosci. 28, 7412-7425. doi: 10.1523/JNEUROSCI.058108.2008

Becker, K., Hohoff, C., Schmitt, B., Christen, H. J., Neubauer, B. A., Sandrieser, T., et al. (2006). Identification of the microdeletion breakpoint in a GLRA1null allele of Turkish hyperekplexia patients. Hum. Mutat. 27, 1061-1062. doi: 10.1002/humu. 9455

Bode, A., and Lynch, J. W. (2013). Analysis of hyperekplexia mutations identifies transmembrane domain rearrangements that mediate glycine receptor activation. J. Biol. Chem. 288, 33760-33771. doi: 10.1074/jbc.M113.513804

Bode, A., and Lynch, J. W. (2014). The impact of human hyperekplexia mutations on glycine receptor structure and function. Mol. Brain 7:2. doi: 10.1186/17566606-7-2

Bode, A., Wood, S. E., Mullins, J. G., Keramidas, A., Cushion, T. D., Thomas, R. H., et al. (2013). New hyperekplexia mutations provide insight into glycine receptor assembly, trafficking, and activation mechanisms. J. Biol. Chem. 288, 33745-33759. doi: 10.1074/jbc.M113.509240

Brams, M., Pandya, A., Kuzmin, D., van Elk, R., Krijnen, L., Yakel, J. L., et al. (2011). A structural and mutagenic blueprint for molecular recognition of strychnine and d-tubocurarine by different cys-loop receptors. PLoS Biol. 9:e1001034. doi: 10.1371/journal.pbio.1001034

Breitinger, H. G., Villmann, C., Becker, K., and Becker, C. M. (2001). Opposing effects of molecular volume and charge at the hyperekplexia site alpha 1(P250) govern glycine receptor activation and desensitization. J. Biol. Chem. 276, 29657-29663. doi: 10.1074/jbc.M100446200

Brune, W., Weber, R. G., Saul, B., von Knebel Doeberitz, M., Grond-Ginsbach, C., Kellerman, K., et al. (1996). A GLRA1 null mutation in recessive hyperekplexia challenges the functional role of glycine receptors. Am. J. Hum. Genet. 58, 989-997.

Buttner, C., Sadtler, S., Leyendecker, A., Laube, B., Griffon, N., Betz, H., et al. (2001). Ubiquitination precedes internalization and proteolytic cleavage of plasma membrane-bound glycine receptors. J. Biol. Chem. 276, 42978-42985. doi: 10.1074/jbc.M102121200

Chung, S. K., Vanbellinghen, J. F., Mullins, J. G., Robinson, A., Hantke, J., Hammond, C. L., et al. (2010). Pathophysiological mechanisms of dominant and recessive GLRA1 mutations in hyperekplexia. J. Neurosci. 30, 9612-9620. doi: 10.1523/JNEUROSCI.1763-10.2010

Du, J., Lu, W., Wu, S., Cheng, Y., and Gouaux, E. (2015). Glycine receptor mechanism elucidated by electron cryo-microscopy. Nature 526, 224-229. doi: 10.1038/nature14853

Emsley, P., Lohkamp, B., Scott, W. G., and Cowtan, K. (2010). Features and development of Coot. Acta crystallographica Section D. Biol. Crystallogr. 66, 486-501. doi: 10.1107/S0907444910007493

Goldberg, A. L. (2003). Protein degradation and protection against misfolded or damaged proteins. Nature 426, 895-899. doi: 10.1038/ nature 02263

\section{FUNDING}

This work was supported by DFG VI586 (CV) NS and GL are supported by the GSLS Würzburg.

\section{ACKNOWLEDGMENTS}

We would like to thank Prof. Dr. K. Schiebel for critical reading of the manuscript and helpful comments. Gudrun Schell and Nadine Vornberger are highly acknowledged for their excellent technical assistance.

Grudzinska, J., Schemm, R., Haeger, S., Nicke, A., Schmalzing, G., Betz, H., et al. (2005). The beta subunit determines the ligand binding properties of synaptic glycine receptors. Neuron 45, 727-739. doi: 10.1016/j.neuron.2005.01.028

Harvey, R. J., Topf, M., Harvey, K., and Rees, M. I. (2008). The genetics of hyperekplexia: more than startle! Trends Genet. 24, 439-447. doi: 10.1016/j.tig.2008.06.005

Hebert, D. N., and Molinari, M. (2007). In and out of the ER: protein folding, quality control, degradation, and related human diseases. Physiol. Rev. 87, 1377-1408. doi: 10.1152/physrev.00050.2006

Hibbs, R. E., and Gouaux, E. (2011). Principles of activation and permeation in an anion-selective Cys-loop receptor. Nature 474, 54-60. doi: 10.1038 /nature10139

Hilf, R. J., and Dutzler, R. (2009). Structure of a potentially open state of a proton-activated pentameric ligand-gated ion channel. Nature 457, 115-118. doi: 10.1038/nature07461

Hoch, W., Betz, H., and Becker, C. M. (1989). Primary cultures of mouse spinal cord express the neonatal isoform of the inhibitory glycine receptor. Neuron 3 , 339-348. doi: 10.1016/0896-6273(89)90258-4

Hooft, R. W., Vriend, G., Sander, C., and Abola, E. E. (1996). Errors in protein structures. Nature 381:272. doi: 10.1038/381272a0

Huang, X., Chen, H., Michelsen, K., Schneider, S., and Shaffer, P. L. (2015). Crystal structure of human glycine receptor-alpha3 bound to antagonist strychnine. Nature 526, 277-280. doi: 10.1038/nature14972

Lape, R., Plested, A. J., Moroni, M., Colquhoun, D., and Sivilotti, L. G. (2012). The alpha1K276E startle disease mutation reveals multiple intermediate states in the gating of glycine receptors. J. Neurosci. 32, 1336-1352. doi: 10.1523/JNEUROSCI.4346-11.2012

Lippincott-Schwartz, J., Roberts, T. H., and Hirschberg, K. (2000). Secretory protein trafficking and organelle dynamics in living cells. Annu. Rev. Cell Dev. Biol. 16, 557-589. doi: 10.1146/annurev.cellbio.16.1.557

Lynch, J. W. (2004). Molecular structure and function of the glycine receptor chloride channel. Physiol. Rev. 84, 1051-1095. doi: 10.1152/physrev.00042.2003

Maksay, G., Biro, T., Laube, B., and Nemes, P. (2008). Hyperekplexia mutation R271L of alphal glycine receptors potentiates allosteric interactions of nortropeines, propofol and glycine with $[3 \mathrm{H}]$ strychnine binding. Neurochem. Int. 52, 235-240. doi: 10.1016/j.neuint.2007.06.009

Moraga-Cid, G., Sauguet, L., Huon, C., Malherbe, L., Girard-Blanc, C., Petres, S., et al. (2015). Allosteric and hyperekplexic mutant phenotypes investigated on an alphal glycine receptor transmembrane structure. Proc. Natl. Acad. Sci. U.S.A. 112, 2865-2870. doi: 10.1073/pnas.1417864112

Pless, S. A., Hanek, A. P., Price, K. L., Lynch, J. W., Lester, H. A., Dougherty, D. A., et al. (2011). A cation-pi interaction at a phenylalanine residue in the glycine receptor binding site is conserved for different agonists. Mol. Pharmacol. 79, 742-748. doi: 10.1124/mol.110.069583

Pless, S. A., and Lynch, J. W. (2009). Ligand-specific conformational changes in the alphal glycine receptor ligand-binding domain. J. Biol. Chem. 284, 15847-15856. doi: 10.1074/jbc.M809343200

Pless, S. A., Millen, K. S., Hanek, A. P., Lynch, J. W., Lester, H. A., Lummis, S. C., et al. (2008). A cation-pi interaction in the binding site of the glycine receptor is mediated by a phenylalanine residue. J. Neurosci. 28, 10937-10942. doi: 10.1523/JNEUROSCI.2540-08.2008 
Polioudaki, H., Kourmouli, N., Drosou, V., Bakou, A., Theodoropoulos, P. A., Singh, P. B., et al. (2001). Histones H3/H4 form a tight complex with the inner nuclear membrane protein LBR and heterochromatin protein 1. EMBO Rep. 2, 920-925. doi: 10.1093/embo-reports/kve199

Rajendra, S., Lynch, J. W., and Schofield, P. R. (1997). The glycine receptor. Pharmacol. Ther. 73, 121-146. doi: 10.1016/S0163-7258(96) 00163-5

Sánchez, R., and Sali, A. (2000). Comparative protein structure modeling. Introduction and practical examples with modeller. Methods Mol. Biol. 143, 97-129. doi: 10.1385/1-59259-368-2:97

Saul, B., Kuner, T., Sobetzko, D., Brune, W., Hanefeld, F., Meinck, H. M., et al. (1999). Novel GLRA1 missense mutation (P250T) in dominant hyperekplexia defines an intracellular determinant of glycine receptor channel gating. J. Neurosci. 19, 869-877.

Schaefer, N., Kluck, C. J., Price, K. L., Meiselbach, H., Vornberger, N., Schwarzinger, S., et al. (2015). Disturbed neuronal ER-Golgi sorting of unassembled glycine receptors suggests altered subcellular processing is a cause of human hyperekplexia. J. Neurosci. 35, 422-437. doi: 10.1523/JNEUROSCI.1509-14.2015

Schaefer, N., Langlhofer, G., Kluck, C. J., and Villmann, C. (2013). Glycine receptor mouse mutants: model systems for human hyperekplexia. Br. J. Pharmacol. 170, 933-952. doi: 10.1111/bph.12335

Schmieden, V., Kuhse, J., and Betz, H. (1993). Mutation of glycine receptor subunit creates beta-alanine receptor responsive to GABA. Science 262, 256-258. doi: $10.1126 /$ science. 8211147

Thompson, J. D., Higgins, D. G., and Gibson, T. J. (1994). CLUSTAL W: improving the sensitivity of progressive multiple sequence alignment through sequence weighting, position-specific gap penalties and weight matrix choice. Nucleic Acids Res. 22, 4673-4680. doi: 10.1093/nar/22.22.4673

Vergouwe, M. N., Tijssen, M. A., Peters, A. C., Wielaard, R., and Frants, R. R. (1999). Hyperekplexia phenotype due to compound heterozygosity for GLRA1 gene mutations. Ann. Neurol. 46, 634-638.

Villmann, C., Oertel, J., Melzer, N., and Becker, C. M. (2009). Recessive hyperekplexia mutations of the glycine receptor alphal subunit affect cell surface integration and stability. J. Neurochem. 111, 837-847. doi: 10.1111/j.1471-4159.2009.06372.x

Wanamaker, C. P., and Green, W. N. (2007). Endoplasmic reticulum chaperones stabilize nicotinic receptor subunits and regulate receptor assembly. J. Biol. Chem. 282, 31113-31123. doi: 10.1074/jbc.M705369200

Yu, R., Hurdiss, E., Greiner, T., Lape, R., Sivilotti, L., and Biggin, P. C. (2014) Agonist and antagonist binding in human glycine receptors. Biochemistry 53, 6041-6051. doi: 10.1021/bi500815f

Conflict of Interest Statement: The authors declare that the research was conducted in the absence of any commercial or financial relationships that could be construed as a potential conflict of interest.

Copyright (c) 2015 Atak, Langlhofer, Schaefer, Kessler, Meiselbach, Delto, Schindelin and Villmann. This is an open-access article distributed under the terms of the Creative Commons Attribution License (CC BY). The use, distribution or reproduction in other forums is permitted, provided the original author (s) or licensor are credited and that the original publication in this journal is cited, in accordance with accepted academic practice. No use, distribution or reproduction is permitted which does not comply with these terms. 